\title{
ESTUDO DE ASPECTOS DA ASSISTENCIA À SAÚDE DA PESSOA IDOSA EM INSTITUIÇÕES NÃO HOSPITALARES DO MUNICIPIO SÃO PAULO, BRASIL
}

\begin{abstract}
BASTIAN, E. M. Estudo de aspectos da assistência à saúde da pessoa idosa em instituiçôes não hospitalares do municipio de São Paulo, Brasil. Rev. Saúde públ., S. Paulo, 11:444-54, 1977.

RESUMO: Foi feito levantamento das instituiçoes para pessoas idosas, exceto em hospitais, no municipio de Sâo Paulo, Brasil, do número de leitos nestas casas, e de aspectos de assistência prestada à saúde das pessoas nelas residentes. Formulou-se a pergunta se o número de leitos influi nesta assistência, no sentido de que instituiçôes com grande número de leitos possa oferecer maior número $e$ variedade dos componentes desta assistência. O resultado da indagação respondeu positivamente a esta pergunta.
\end{abstract}

Unitermos: Geriatria. Velhos. Casas para velhos. Assistência médica.

\section{N T RODUCAO}

Em paises com uma taxa elevada de população idosa, a problemática geriátrica e gerontológica é impossivel de ser ignorada. Taxas de $17,0 \%$ como na Dinamarca, de $16,5 \%$ na Suiça e de $10,4 \%$ na Suécia ${ }^{4}$, de população com mais de 60 anos, fazem com que os recursos assistenciais para este grupo etário estejam desenvolvidos.

Em comparação com estes paises, o Brasil é um país de jovens. Estimativas da população brasileira pelo Instituto Brasileiro de Geografia e Estatística (IBGE) ${ }^{1}$, para o ano 1970, indicaram uma taxa entre 4 e $5 \%$ para pessoas de mais de 60 anos.

Para o municipio de São Paulo há estimativas para 1980 de $5,3 \%$ para este gru- po. Em números absolutos, o municipio de São Paulo terá em 1980 , 495.300 pessuas com mais de 60 anos. Desta população, uma parte deverá ser encontrada vivendo na comunidade, outra em hospitais - gerais e geriátricos - outra em estabelecimentos que abrigam pessoas idosas em regime de internato, com maior ou menor capacidade de assistência à saúde dos seus residentes.

Encontramos na literatura especifica uma pesquisa feita nos Estados Unidos, pelo Governo Federal daquele pais ${ }^{8}$, sobre recursos assistenciais para a saúde da pessoa idosa em instituições, exceto hospitais, e sua relação com o número de leitos nestes estabelecimentos. Esta pesquisa revelou que, quanto mais leitos, mais completa fora a

* Do Departamento de Prática de Saúde Pública da Faculdade de Saúde Pública da USP, Av. Dr. Arnaldo, 715 - São Paulo, SP - Brasil. 
BASTIAN, E. M. Estudo de aspectos da assistência à saúde da pessoa idosa em instituições não hospitalares do município de São Paulo. Brasil. Rer. Saúde públ., S. Paulo, 11:444-54, 1977.

equipe assistencial a serviço da pessoa abrigada.

Townsend citado por Greenwald \& Linn ${ }^{\circ}$, investigando 173 internatos na Inglaterra, relacionou a qualidade de assistência com () tamanho dos internatos. Beatty \& Bullok, também citados por Greenwald \& Linn, numa pesquisa em 80 internatos em St. Louis, Estados Unidos, encontraram uma associação positiva entre 0 tamanho da instituição e a avaliaçāo favorável da assistência prestada. E os estudos de Greenwald \& Linn ${ }^{6}$ sobre intercorrelação de serviços confirmaram os resultados de Siroccos, ou seja, quanto mais leitos, mais completa a equipe de pessoal assistencial à saúde.

Achamos este enfoque de interesse para nosso estudo como referência para a explicação das falhas nesta assistência em nosso meio ${ }^{5}$.

Visou-se com este estudo:

- identificar a número de instituições não hospitalares para a pessoa idosa, no município de São Paulo, e o número de leitos nestas instituições, $\mathrm{e}$

- verificar se as instituições com grande número de leitos oferecem aos seus residentes assistência à saúde mais completa. Isto, no sentido de maior número de componentes desejáveis desta assistência, do que instituições com poucos leitos.

Considerou-se como componentes de assistência desejáveis:

- médico de tempo integral;

- odontólogo;

- enfermeira de tempo integral;

- serviço de enfermagem noturno, prestado por enfermeira ou auxiliar de enfermagem;

- nutricionista ou dietista;

- assistente social;

- terapia ocupacional;
- assistência cultural;

- assistência recreacional;

- assistência religiosa.

\section{MATERIAL E MÉTODOS}

$O$ objeto da pesquisa foram as instituições não hospitalares do município de São Paulo que aceitam em regime de internato pessoas idosas.

As fontes desses estabelecimentos foram. as listagens da Secretaria do Bem-Estar Social da Prefeitura do Município de São Paulo, da Secretaria da Promoção Social do Estado de São Paulo e da Federação de Obras Sociais (FOS), esta fornecida pelo Serviço Social do Comércio (SESC).

Foram elaborados uma ficha de identificação do número de leitos nas instituições e um formulário para o inquérito em entrevistas pessoais com os dirigentes destas casas.

Após dois pré-testes deste formulário e mudificações que se mostraram convenientes, foram preparados dois entrevistadores que realizaram o inquérito, conseguindo, após dois retornos, rendimento de $100 \%$ do universo de 44 instituições.

\section{RESULTADOS E DISCUSSÃO}

Apresentamos nas Tabelas 1 a 6 os resultados obtidos. Reunimos as instituiçōes conforme o número de leitos. O tamanho dos grupos foi escolhido de acordo com o pressuposto de que encontraríamos maior número de instituições com poucos leitos, 1) que no inquérito foi confirmado.

$\mathrm{Na}$ Tabela 1 apresentamos a distribuição das variantes da Assistência Médica nas instituições. Esta distribuição mostra que todos os grupos dispõem de médico que visita regularmente os residentes. Destaca-se o grupo B com $91,6 \%$, enquanto os grupos $C, D, E$ e $F$ estão representados com $66,6 \%$, seguidos pelo grupo A, com $62,6 \%$. 
BASTIAN, E. M. Estudo de aspectos da assistência à saúde da pessoa idosa em instituições não hospitalares do município de São Paulo, Brasil. Rev. Saúde públ., S. Paulo, 11:444-54, 1977 .

TABELA 1

Assistêncla médica segundo o número de leitos nas instituições não hospitalares do Município de São Paulo.

\begin{tabular}{|c|c|c|c|c|c|}
\hline & \multirow{2}{*}{$\begin{array}{r}\text { Assistência } \\
\text { médica }\end{array}$} & \multicolumn{3}{|c|}{ Tem médico } & \multirow{2}{*}{$\begin{array}{c}\text { Năo tem } \\
\text { médico } \\
(\%)\end{array}$} \\
\hline $\begin{array}{l}\text { No de } \\
\text { Jeitos }\end{array}$ & & $\begin{array}{c}\text { Tempo } \\
\text { integral } \\
(\%)\end{array}$ & $\begin{array}{l}\text { Visitas } \\
\text { regulares } \\
(\%)\end{array}$ & $\begin{array}{c}\text { Só em caso } \\
\text { de doença } \\
(\%)\end{array}$ & \\
\hline (A) $<30$ & 8 & $\begin{array}{c}12,5 \\
(1)\end{array}$ & $\begin{array}{l}62,5 \\
(5)\end{array}$ & - & $\begin{array}{l}25,0 \\
(2)\end{array}$ \\
\hline $30-59$ & 12 & - & $\begin{array}{l}91,6 \\
(11)\end{array}$ & - & $\begin{array}{l}8,3 \\
\text { (1) }\end{array}$ \\
\hline$\frac{60-}{(\mathrm{C})} 99$ & 12 & - & $\begin{array}{l}66,7 \\
(8)\end{array}$ & $\begin{array}{l}25,0 \\
(3)\end{array}$ & $\begin{array}{l}8,3 \\
(1)\end{array}$ \\
\hline $\begin{array}{c}100-199 \\
\text { (D) }\end{array}$ & 6 & $\begin{array}{l}16,6 \\
\text { (1) }\end{array}$ & $\begin{array}{c}66,7 \\
(4)\end{array}$ & 一 & $\begin{array}{l}16,6 \\
(1)\end{array}$ \\
\hline $\begin{array}{c}200-499 \\
(E)\end{array}$ & 3 & $\begin{array}{l}33,3 \\
(1)\end{array}$ & $\begin{array}{l}66,7 \\
(2)\end{array}$ & - & - \\
\hline$\underset{(F)}{500 \mathrm{e}}+$ & 3 & $\begin{array}{l}33,3 \\
(1)\end{array}$ & $\begin{array}{l}66,7 \\
(2)\end{array}$ & - & - \\
\hline Total & 44 & $\begin{array}{l}9 ; 0 \\
(4)\end{array}$ & $\begin{array}{l}72,7 \\
(32)\end{array}$ & $\begin{array}{l}6,8 \\
(3)\end{array}$ & $\begin{array}{c}11,3 \\
(5)\end{array}$ \\
\hline
\end{tabular}

No total geral, somente $9,0 \%$ das casas contam com médico de tempo integral. A maior representação tem o médico que visita os residentes regularmente, isto em $72,7 \%$ das instituições. Em 6,8\% o médico só atende em caso de doença, e $11,3 \%$ não tem médico que atenda.

Siroccos, numa pesquisa sobre o tipo de assistência médica prestada à população idosa em 19.476 internatos nos Estados Unidos (o universo), verificou que aproximadamente $4 \%$ dos internatos contaram com médico em regime de tempo integral.

Em segundo lugar apareceu a visita do médico à instituição em intervalos regulares $(34 \%)$. Em $3 \%$ das casas não houve assistência médica.

Comparando a situação das instituições de São Paulo com as dos Estados Unidos,
(Sirocco ${ }^{8}$ ) dentro das limitações de comparar dados de um município com os de um pais, observa-se que em São Paulo $9 \%$ das instituiçōes têm médico de tempo integral, enquanto nos Estados Unidos somente $4 \%$. Em São Paulo, 72,7\% recebem visitas regulares do médico contra $34 \%$ das instituiçōes nos Estados Unidos. A taxa das casas que não providenciam qualquer assistência médica, porém, é maior em São Paulo, 11,3\%; nos Estados Unidos é de $3 \%$.

A existência ou não de uma Assistência Odontológica nas instituições, pode ser observada na Tabela 2 .

Nos grupos E e F, $100 \%$ das instituições dispõem de dentista para atender aos problemas dentários nos residentes; no grupo 
BASTIAN, E. M. Estudo ce aspectos da assistência à saúde da pessoa idosa em instituições não hospitalares do município de São Paulo, Brasil, Rev. Saúde pabl., S. Paulo, 11:444-54, 1977.

A não há nenhuma assistência odontológi- fica que no total geral somente $31,8 \%$ ca; no grupo $C$, existe somente em $16,6 \%$, possuem assistência odontológica e $68,1 \%$ e nos grupos B e D em 33,3\%. Isto signi- das casas não a possuem.

T A B ELA 2

Assistência odontológica segundo o número de leitos nas instituições não hospitalres no nıunicípio de São Paulo.

\begin{tabular}{|c|c|c|c|}
\hline \multirow[b]{2}{*}{$\begin{array}{l}\text { No de } \\
\text { leitos }\end{array}$} & \multirow{2}{*}{$\underbrace{\begin{array}{r}\text { Assistência } \\
\text { odontológica }\end{array}}_{\mathrm{N} \text { Instit. }}$} & \multicolumn{2}{|c|}{ Tem dentista } \\
\hline & & $\operatorname{Sim}(\%)$ & Não (\%) \\
\hline$\underset{(\mathrm{A})}{<30}$ & 8 & & $\begin{array}{c}100,0 \\
(8)\end{array}$ \\
\hline $\begin{array}{l}30-59 \\
\text { (B) }\end{array}$ & 12 & $\begin{array}{l}33,3 \\
(4)\end{array}$ & $\begin{array}{l}66,7 \\
(8)\end{array}$ \\
\hline $60-99$ & 12 & $\begin{array}{l}16,6 \\
(2)\end{array}$ & $\begin{array}{l}83,3 \\
(10)\end{array}$ \\
\hline $100-199$ & 6 & $\begin{array}{l}33,3 \\
(2)\end{array}$ & $\begin{array}{l}66,7 \\
(4)\end{array}$ \\
\hline $\begin{array}{c}200-499 \\
(\mathrm{E})\end{array}$ & 3 & $\begin{array}{c}100,0 \\
(3)\end{array}$ & - \\
\hline $500 \underset{(F)}{\mathrm{e}}+$ & 3 & $\begin{array}{c}100,0 \\
(3)\end{array}$ & - \\
\hline Total & 44 & $\begin{array}{l}31,8 \\
(14)\end{array}$ & $\begin{array}{l}68,1 \\
(30)\end{array}$ \\
\hline
\end{tabular}

Encontram-se na Tabela 3 as categorias de pessoal para a Assistência de enfermagem e sua distribuição nos 6 grupos de instituições.

Em todos os grupos existe a enfermeira. $\mathrm{Na}$ melhor situação encontra-se o grupo $\mathrm{E}$, onde todas as enfermeiras são de tempo integral; nos grupos A e B o são $50,0 \%$ e $66,7 \%$, respectivamente. $O$ único grupo com enfermeira de tempo parcial é o grupo C, com $8,3 \%$ das instituições deste grupo.

A auxiliar de enfermagem está presente em $66,7 \%$ das casas do grupo $\mathrm{F}$; a atendente em $33,3 \%$ do grupo $D$.

O total geral mostra que se conta com a enfermeira em $52,2 \%$ das instituições; a auxiliar está presente em $27,2 \%$ e a atendente em $13,6 \%$. Somente en dois grupos, $\mathrm{B}$ e $\mathrm{C}$, há exclusivamente leigos para cuidar dos residentes.

No estudo relatado por Siroccos, nos Estados Unidos, há no total $54 \%$ de enfermeiras; em São Paulo encontramos 52,2\%; são $19 \%$ de auxiliares de enfermagem nos Estados Unidos, contra $27,2 \%$ entre nús, e $11 \%$ de atendentes contra $13,6 \%$ em São Paulo.

A exigência de Leeds \& Shore ", de que no interesse de cuidados de alta qualidade, haja a enfermeira as 24 horas do dia, está melhor cumprida em São Paulo, onde há $50 \%$, do que nos Estados Unidos, onde são somente $16 \%$. 


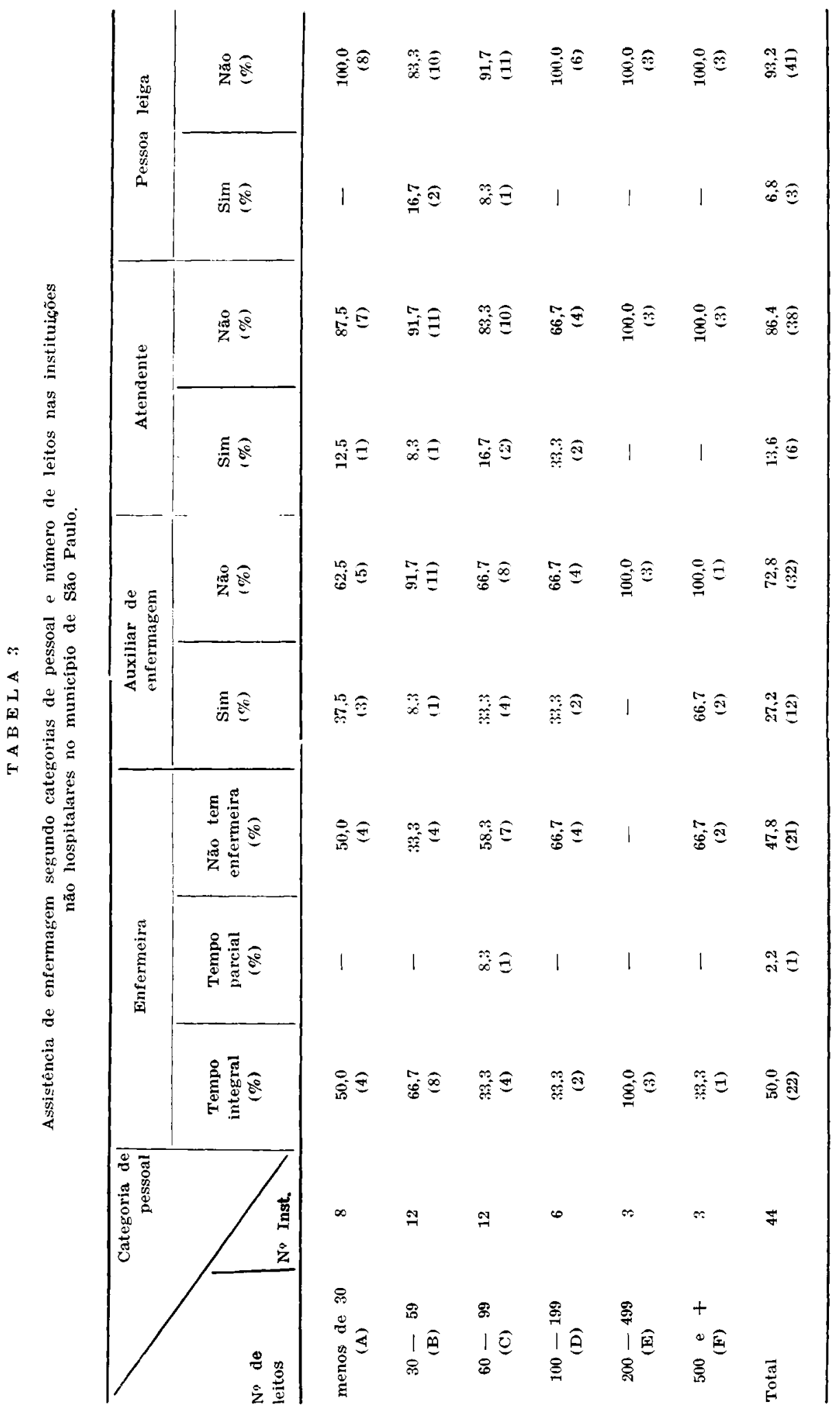


BASTIAN, E. M. Estudo de aspectos da assistência à saúde da pessoa idosa em instituições não hospitalares do municipio de Săo Paulo, Brasil. Rev. Saúde públ., S. Paulo, 11:444-54, 1977.

Esta percentagem mais elevada em São Paulo poderá refletir uma prática em nosso meio, de delegar algumas atividades médicas à enfermeira, viável neste tipo de instituições, nas quais os residentes necessitam principalmente uma competente supervisão da saúde.
Os informantes de todas as 44 instituiçōes afirmam ter serviço de enfermagem noturno. Verifica-se na Tabela 4 as categorias de pessoal que prestam este serviço.

Conta-se para o serviço nuturno com enfermeira de tempo integral em $41,6 \%$ das casas do grupo B, e em $37,5 \%$ do

T A B ELA 4

Serviço de enfermagem noturno segundo categoria de pessoal, número de leitos e instituiçõcs não hospitalares do Município de São Paulo.

No de
leitos

grupo $\mathrm{A}$, os dois grupos de menor número de leitos. O serviço noturno é prestado por auxiliares de enfermagem em $100 \%$ das instituições do grupo F, e em $66,7 \%$ do grupo E. A atendente como único pessoal noturno está no grupo $B$, com $50 \%$, seguido pelo grupo A, com $37,5 \%$. Os grupos $B$ e C contam somente com leigos, com $25 \%$ e $16,6 \%$ respectivamente.

O total geral mostra uma presença com $25 \%$ da enfermeira, $40,9 \%$ da auxiliar de enfermagem, $22,7 \%$ da atendente e $11,3 \%$ de pessoal leigo, na prestação de serviços de enfermagem noturno.

No seu Relatório de Atividades ${ }^{3}$, a maior das instituições, com 853 leitos, informa que seu serviço de enfermagem tem como objetivo zelar diuturnamente pela saúde dos internados, de comum acordo com a orientação médica. Sua equipe de trabalho é composta de duas enfermeiras de alto padrão, sendo uma supervisora geral do serviço de enfermagem, dois auxiliares de enfermagem e 26 atendentes. Dois 
BASTIAN, E. M. Estudo le aspectos da assistência à saúde da pessoa idosa em instituiçóes não hospitalares do municip1o de São Paulo, Brasil. Rer. Saúdz públ., S. Paulo, 11:444.54, 1977 .

funcionários sāo sempre escalados para 0 plantão noturno.

O Serviço de Nutrição e Dietética é deficiente nas instituiçōes. Encontramos os respectivos nutricionistas em uma instituiçäo do grupo $\mathrm{F}$, representando $33,3 \%$ deste grupo. Uma casa do grupo E está só temporariamente sem nutricionista. São duas instituições dos grupos com maior número de leitos. Os dois grupos do outro extremo, isto é, com poucos leitos, A e B contam com dietista. No total geral, o profissional está presente somente em $6,8 \%$ dos estabelecimentos.

Os dirigentes das instituiçōes, na maioria, informam que o médico prescreve o tipo de alimento ou dieta para os residentes. A pessoa responsável pela cozinha - que varia de enfermeira à pessoa leiga - prepara e, conforme qualificação, supervisiona o cardápio.

O Assistente Social está presente em todos os grupos, sendo $100 \%$ nos grupos E e F, 50\% nos grupos B, C e D e 37,5\% no grupo $\mathrm{A}$.

Nos grupos B, C e D, quando não há assistente social, o que ocorre em $33,3 \%$, $8,3 \%$ e $33,3 \%$, respectivamente, os residentes são encaminhados a serviços oficiais e particulares. Do grupo A, $62,5 \%$ e do grupo C, $41,7 \%$ das casas não tomam qualquer providência para que seus residentes recebam esta assistência.

No total geral, $54,5 \%$ das casas dispóem em seu quadro de pessoal, do assistente social. São feitos encaminhamentos em $15,9 \%$, e $29,6 \%$ estão sem providências.

A situação da Terapia Ocupacional* nas instituições é a seguinte: a maioria das casas diz propiciar alguma forma de terapia ocupacional a seus residentes. Os dois grupos de maior número de leitos, grupos $E$ e F, fazem-se presentes com $100 \%$. Seguem os grupos B e C com $83,3 \%, 0$ grupo D com $66,7 \%$ e o grupo A com $50 \%$.

No total geral, $77,2 \%$ informam oferecer terapia ocupacional aos idosos.

Deve ser esclarecido, porém, que somente 3 instituições, pertencentes aos grupos com grande numero de leitos, tratam da presença de un terapeuta ocupacional profissional. Nos demais, os responsáveis pela casa dizem incentivar a ocupação dos seus residentes para que estes se dediquem a trabalhos manuais. $O$ valor terapêtico destas atividades, evidentemente, depende da capacitação das pessoas que possam dar orientação a estas ocupaçōes.

Os recursos disponiveis para dar uma Assistência Cultural** aos residentes, podem ser verificados na Tabela 5 .

Entre as atividades culturais oferecidas se destacam as conferências, presentes em todos os grupos de instituiçōes: em todas as dos grupos E e F, com 58,3\% no grupo $C$ e com $50 \%$ no grupo D.

Seguem em frequiência as exposiçōes e os concertos. Os primeiros estão mencionados com $66,7 \%$ no grupo E, $41,6 \%$ no grupo C. Os concertos estão presentes em todo o grupo $F$ e em $33,3 \%$ nos grupos $D$ e $E$. Cursos e teatros têm pouca representação.

No total dos recursos culturais figuram com a maior percentagem, $43,1 \%$, as conferências. Em seguida as exposiçōes, com $31,8 \%$, concertos com $25,0 \%$, cursos e teatros com $6,8 \%$ cada.

É lamentável que somente 3 instituiçōes propiciem cursos para os residentes. Sabese que a pessoa idosa necessita do desafio intelectual para continuar a se desenvolver e realizar, capacidade que é comprovada no idoso enquanto estimulado e orientado.

\footnotetext{
* Terapia Ocupacional "é o tratamento de problemas de saúde, mentais ou físicos, por meio de trabalho destinado a distrair a mente ou corrigir um deteminado defeito fisico, ou uma atividade criativa que possa promover a recuperação ou reabilitação" (Sirocco 8).

* Enquadramos sob o tópico Assistência Cultural o oferecimento de atividades que promoram a formação ou o aperfeiçoamento do intelecto, das práticas e da sensibilidade estética do individuo.
} 


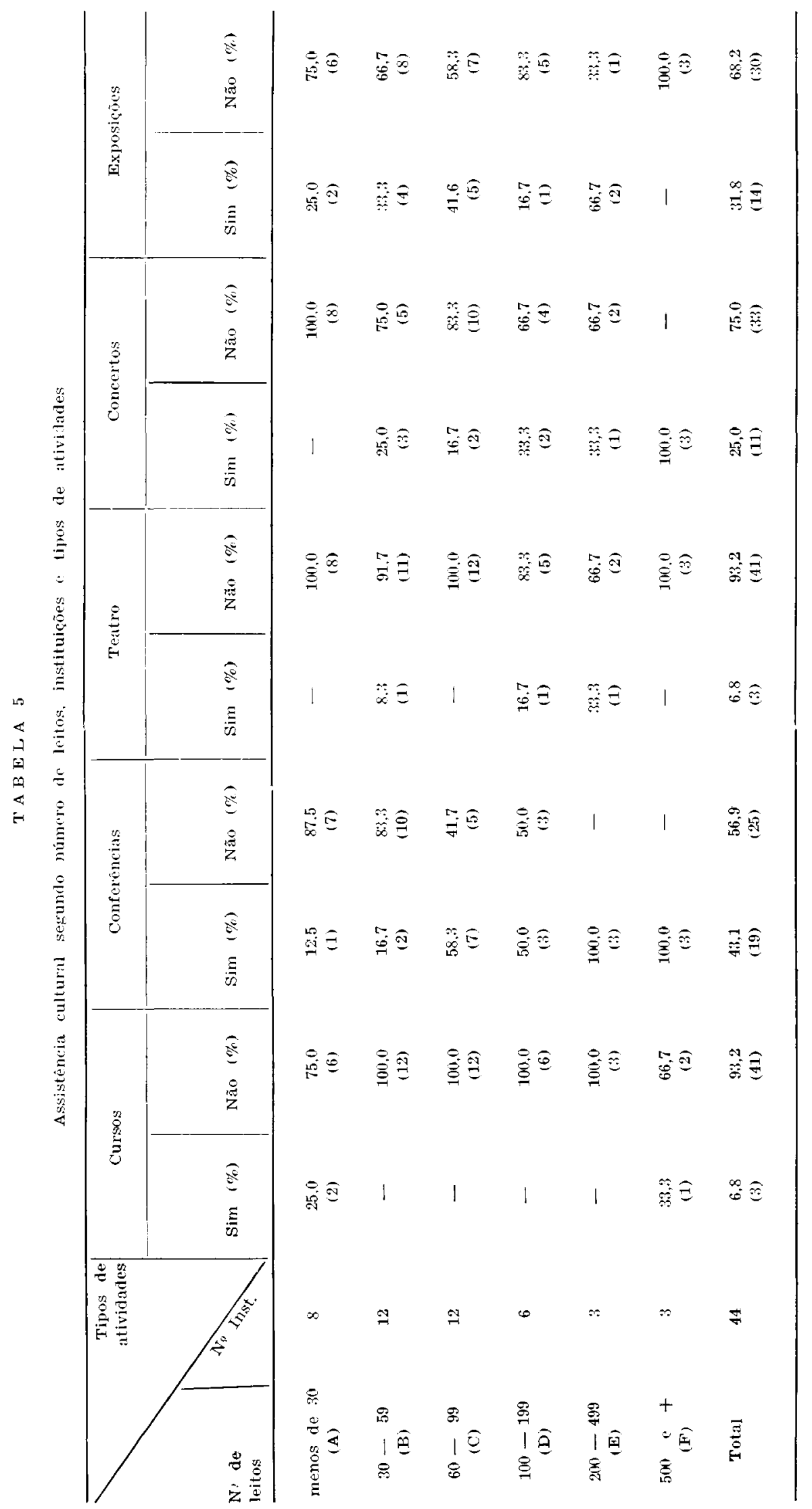


EASTIAN, E. M. Estudo de aspectos du assistoncla à saúde da pessoa idosa em instituiģoes não hospitalares do município de São Paulo. Brasil. Rer. Saude pübl., S. Paulo. 11:444-54. 1977 .

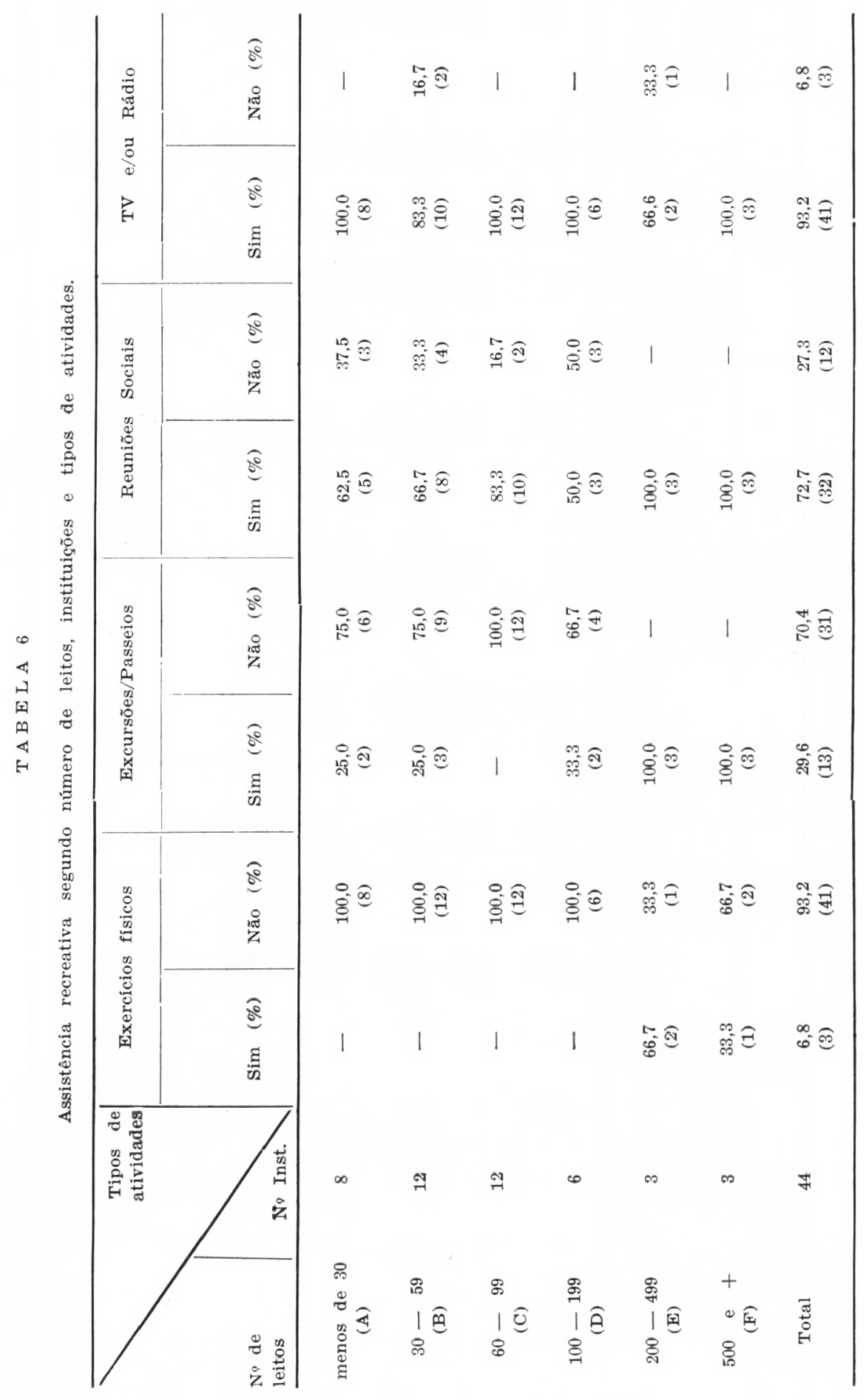


BASTIAN, E. M. Estudo de aspectos da assistência à saúde da pessoa idosa em instituições não hospitalares do município de São Paulo, Brasil. Rev. Saúde públ., S. Paulo, 11:444-54, 1977 .

Vè-se ainda na Tabela 6 a distribuição das atividades oferecidas aos residentes para sua Recreação*.

Os dois recursos predominantes para fins recreativos em quase todos os grupos são a TV e o rádio, mencionados em todas as instituições dos grupos A, C, D e F.

En segundo lugar destacam-se as renniões sociais, presentes na totalidade dos grupos E e $F$, seguidos por $83,3 \%$ no grupo $C, 66,7 \%$ no grupo $B, 62,2 \%$ e $50,0 \%$ nos grupos A e D, respectivamente. O terceiro lugar ocupam as excursões e passeios, também presentes em todas as casas dos grupos E e F, com 33,3\% no grupo D, em $25 \%$ dos grupos A e B, e sem representação no grupo $C$. Não são promovidos exercícios físicos nos grupos A, B, C e D. São oferecidos em $66,6 \%$ do grupo E e em $33,3 \%$ do grupo F.

No total geral, TV e rádio estão em primeiro lugar, com $93,2 \%$; en segundo lugar vêm as reuniões sociais com $72,7 \%$; seguem as excursões e passeios com $29,6 \%$ e, por último, os exercícios físicos, com $6,8 \%$.
Há um relato do Departamento de realizaçōes culturais, de uma instituição com grande número de leitos (380 leitos), que diz: "Aos nossos residentes foi oferecido ampla e variada programação... com a colaboração de artistas, grupos folclóricos de "ballet", orquestras sinfônicas... O nosso coral. . composto exclusivamente por residentes da casa, apresentou-se... inclusive em realizações externas. Introduzimos em 1974 cursos de ginástica, sob orientação médica, com a participação de elevado número de residentes nossos".

Os grupos C, D, E e F em sua totalidade oferecen alguma forma de Assistência Religiosa, variando somente os credos dos residentes. $O$ grupo $A$ está representado com $87,5 \%$ e o grupo B com $83,3 \%$. O total geral chega a $93,2 \%$. Mais da metade, ou 27 das 44 instituições, está mantida por grupos religiosos, o que explica esta alta percentagem.

Classificamos as instituições em categorias I a IV. Atribuimos pesos 3,2 e 1 aus componentes de assistência que postulamos como desejáveis, 3 representando

T A B E A 7

Classificação das instituições, número e percentagem nas categorias de assistência

\begin{tabular}{|c|c|c|c|}
\hline \multirow{2}{*}{$\begin{array}{l}\text { Categoria de } \\
\text { assistência }\end{array}$} & \multirow{2}{*}{ Classificação } & \multicolumn{2}{|c|}{ Instituições } \\
\hline & & Ne & $\%$ \\
\hline I & ótimo & 6 & 13,6 \\
\hline II & satisfatório & 12 & 27,2 \\
\hline III & regular & 16 & 36,4 \\
\hline IV & insuficiente & 10 & 22,7 \\
\hline Total & & 44 & 99,9 \\
\hline
\end{tabular}

* Como recreacão entendemos a renovação de corpo e mente por meio de formas de relaxa- . mento, divertimento e de atividades de livre criatividade. 
BASTIAN, E. M. Estudo de aspectos da assistência à saúde da pessoa idosa em instituições não hospitalares do município de São Paulo, Brasil. Rev. Saúde públ., S. Paulo, 11:444-54, 1977.

a importância maior, 2 a média e 1 a importância menor.

A Tabela 7 mostra que o maior número de instituições, ou seja, 16 pertencem à categoria III, consideradas "regulares"; 10 figuram na categoria IV, "insuficiente"; 12 na categoria II, sendo "satisfatórias". Somente 6 classificam-se como "ótimas", na categoria 1 . Isto significa que 18 instituições, ou $40,9 \%$, pertencentes às duas categorias melhor classificadas, podem ser recomendadas. Vinte e seis casas, ou $59,1 \%$, não representam um bom padrão quanto à assistência à saúde que oferecem aos residentes.

\section{CON CLUSOES}

Dos resultados desta pesquisa concluimos:

a) Existem no município de São Paulo 44 instituições não hospitalares que abrigam pessoas idosas. $O$ número de leitos se distribui entre a menor com 12 e a maior instituição com 853 leitos. O número total de leitos é de 5.130 .

b) Podemos responder, na afirmativa à suposição contida no segundo objetivo, que as instituições com grande número de leitos contam com maior número dos componentes de assistência que consideramos desejáveis.

\section{RSPU-B/377}

BASTIAN, E. M. (Health care for the elderly - a study on some aspects of non hospital institutions in the city of $S$. Paulo, Brazil] Rev. Saude públ., $S$. Paulo, 11:444-54, 1977.

ABSTRACT: A survey was done in institutions for elderly people, except in hospitals, in the county of São Paulo, on the number of beds in these homes and aspects of health care offered to their residents. The question was raised whether the number of beds had an influence on health care, in the way that homes with a larger number of beds offered a greater number and more variety of the components of this care. The outcome of the research answered this question positively.

UnITERMS: Geriatrics. Aged. Homes for the aged. Medical assistance.

\section{REFERENCIAS BIBLIOGRAFICAS}

1. ANUARIO ESTATISTICO DO BRASIL. (Instituto Brasileiro de Geografia e Estatística) Rio de Janeiro, 1971.

2. BOLETIM INFORMATIVO DA SOCIEDADE RELIGIOSA E BENEFICENTE ISRAELITA NOSSO LAR. São Paulo (21), jul. 1974

3. COELHO DE FARIA, C. - Relalório de atividades. Departamento Dom Pedro II. São Paulo, Santa Casa de Misericórdia 1971.

4. DEMOGRAPHIC YEARBOOK. (United Nations) New York, 1970.

5. FERRARI. M. A. C. - Geriatria: aspectos de educasão em saúde $e$ terapia ocupacional. São Paulo, 1975. (Mono- grafia de Mestrado - Faculdade de Saúde Pública, USP).

6. GREENWALD, S. R. \& LINN, M. W. Intercorrelation of data on nursing homes. Gerontologist, 2:337-40, 1971.

7. LEEDS, M. \& SHORE, H. - Geriatric institutional management. New York, Putnam's Sons, 1964.

8. SIROCCO, A. Services and activities offered to nursing home residents, United States - 1968. Rockville, Md., National Center for Health Statistics. 1972. (Vital and Health Statistics, series 12 , no 17 ).

Recebido para publicação em 11/03/1977. Aprovado para publicagão em 28/03,197\%. 